ORIGINAL

\title{
PREVALENCIA DE TRATAMIENTO DE LA INFECCIÓN TUBERCULOSA EN UNA PRISIÓN PROVINCIAL
}

\author{
Vicente Martín, Margarita Brugos e Irene Valcarcel \\ Área de Medicina Preventiva y Salud Pública. \\ Escuela de Enfermería. Universidad de León.
}

\section{RESUMEN}

Fundamentos: La tuberculosis en prisión es un grave problema de salud pública. En aquellos centros con elevadas prevalencias de coinfección por $M$. tuberculosis e infección VIH el tratamiento de la infección tuberculosa puede ser una importante herramienta de prevención y control. Conocer la prevalencia de infección tuberculosa, el acceso y la adherencia al tratamiento de la infección tuberculosa en población reclusa sin antecedentes de tratamiento de enfermedad o infección tuberculosa puede ser de gran interés para valorar la efectividad de esta medida.

Métodos: Se realizó un estudio transversal en la población ingresada a fecha 28 de junio de 1999 en un Centro Penitenciario con un programa de prevención y control de la tuberculosis basado en la terapia directamente observada (TDO) de los enfermos, eil el diagnóstico precoz y el tratamiento de la infección tuberculosa. El tratamiento de la infección tuberculosa se prescribió tras consejo médico individualizado consensuándose con el paciente la modalidad del mismo (TDO, pauta diaria o bisemanal). Se revisaron las historias clínicas de los pacientes ingresados para conocer la prevalencia de pacientes infectados por $M$. tuberculosis, de los que habian finalizado y de los que se encontraban realicando tratamiento de la infección tuberculosa. Se realizó el test de Eidus-Hamilton en el caso de los pacientes en tratamiento frente a la infección tuberculosa para valorar su cumplimiento.

Resultados: De los 219 internos, $127(58 \%)$ presentaban criterio de infección tuberculosa. Se indicó tratamiento de la infección tuberculosa en 113 pacientes de los que 29 no aceptaron $(25,7 \%)$. De los 84 pacientes que iniciaron tratamiento de la infección tuberculosa, $22(26,2 \%)$ lo abandonaron, $39(46,4 \%)$ habian finalizado el tratamiento de la infección tuberculosa, y 23 $(27,4 \%)$ se encontraban realizándola. De estos últimos el $95,7 \%$ resultaron positivos al test de Eidus-Hamilton. El $48 \%$ de los infectados por $M$. tuberculosis, o habían finalizado o estaban realizando correctamente el tratamiento de la infección tuberculosa.

Conclusión: La prevalencia de infección tuberculosa observada en este colectivo es muy elevada. Se han observado aceptables niveles de acceso y adhcrencia al tratamicnto de la infección tuberculosa lo que se traduce en una elevada cobertura de tratamiento de la infección tuberculosa en la población estudiada.

Palabras clave: Tuberculosis. Prisión.

Correspondencia:

Vicente Martín Sánchez

Área de Medicina Preventiva y Salud Pública.

Departamento de Fisiología. Facultad de Veterinaria.

Campus de Vegazana s/n - 24071 León

Correo electrónico: dfivms@unileon.es

\section{ABSTRACT
Prevalence of Treatment of Tuberculosis Infection in a Provincial Prison

Background: Tuberculosis in prisons is a serious public health problem. In those penitentiaries with a high prevalence of co-infection by $M$. tuberculosis and HIV infection, the treatment of the tubercular infection can be an important tool for prevention and control. Ascertaining the prevalence of tubercular infection, the access and adherence to the treatment of tubercular infection in a prison population without a history of treatment of tubercular disease or infection could be of great interest in order to assess the efficacy of this measure.

Methods: A cross study was made of the population confined at 28 June 1999 in a Penitentiary with a programme of prevention and control of tuberculosis, based on the directly observed therapy (DOT) of the patients, on early diagnosis and the treatment of the tubercular infection. The treatment of the tubercular infection was prescribed following individualised counselling, reaching an agrecment with the patient as to the modality of treatment (DOT, daily or twice-weekly check-up). The clinical histories of the patients were reviewed in order to determine the prevalence of patients infected by $M$. tuberculosis, of those who had completed treatment and of those who were still undergoing treatment of tubercular infection. The EidusHamilton test was performed in the case of patients under treatment for iubercular infection in order to evaluate compliance.

Results: Of the 219 inmates, $127(58 \%)$ presented criteri of tubercular infection. Treatment of the tubercular infection was indicated in 113 people of whom 29 refused $(25.7 \%)$. Of the 81 patients who initiated treatment for their tubercular infection, 22 $(26.2 \%)$ abandoned it, $39(46.4 \%)$ had finalised the treatment, and $23(27.4 \%)$ were still undergoing treatment. Of the latter, $95.7 \%$ showed positive to the Eidus-I Iamilton test. $48 \%$ of those infected by $M$. tuberculosis, either had finalised or were undergoing the treatment against tubercular infection correctly.

Conclusion: The prevalence of tubercular infection observed in this group is very high. Acceptable levels of access and adherence to the treatment of tubercular infection have been observed, which translates into a high level of treatment coverage of this infection in the population studied.

Key Words: Tuberculosis. Prison. 


\section{INTRODUCCIÓN}

La tuberculosis (TB) es una enfermedad de gran relevancia en el medio penitenciario, por convivir en el mismo personal de riesgo para la infección y la enfermedad tuberculosa y por la facilidad que el vivir en una institución cerrada supone para la transmisión del bacilo de Koch, por lo que su prevención y control merece especial atención ${ }^{1}$. Entre las actividades más eficaces en este campo está el tratamiento de la infección tuberculosa latente (TIT) ${ }^{2}$, que en el medio penitenciario, dadas las altas prevalencias de coinfección por virus de la inmunodeficiencia humana (VIH) y $M$. tuberculosis ${ }^{3}$, presenta un importante valor añadido ${ }^{4}$.

Sin embargo, la efectividad del TIT viene penalizada por un limitado acceso al mismo de los pacientes en los que está indicado y un elevado número de abandonos por diversas razo$n e s^{5}$. En el caso de la población reclusa se vienen a añadir problemas específicos entre los que destaca su gran movilidad ${ }^{6}$.

A pesar de las dificultades asociadas a la efectividad del TIT, diversos estudios han observado que ésta puede ser mejorada ${ }^{7,8}$. El objetivo del presente trabajo es conocer la prevalencia de infección tuberculosa, el acceso y la adherencia al TIT en población reclusa sin antecedentes de tratamiento de enfermedad o infección tuberculosa.

\section{SUJETOS Y MÉTODOS}

Se llevó a cabo un estudio transversal de prevalencia que incluyó a toda la población ingresada en el centro penitenciario el día 28 de junio de 1999. Se consultaron las historias clínicas de todos los sujetos, para recoger variables demográficas (edad, sexo, nacionalidad, residencia, formación académica, ocupación laboral, antecedentes de ingresos previos en prisión, etc.) y clínicas (antecedentes de enfermedad e infección tuberculosa, resultado de las pruebas de la tuberculina, infección VIH, factores de riesgo para la infección VIH, etcétera).

El promedio de internos del CP era de 200 a 250 , con unos 500 ingresos y excarcelaciones anuales. La mayoría de la población era penada y mayor de 21 años. Desde agosto de 1990 se llevaba a cabo el Programa de Prevención y Control de la Tuberculosis de la Dirección General de Instituciones Penitenciarias ${ }^{9}$, consistente fundamentalmente en la búsqueda activa y pasiva de casos, la terapia directamente observada en los enfermos (TDO) y el TIT con isoniacida (INH) en los infectados. A todo sujeto que ingresaba se le ofrecía practicar la prueba de la tuberculina con 2 UT de PPD RT23 según la técnica de Mantoux, con lectura a las 48-96 horas, considerándose infectados todos aquellos que presentaban una induración superior o igual a $5 \mathrm{~mm}$ en el caso de los no vacunados con $\mathrm{BCG}$ o infectados por VIH, y superior o igual a $15 \mathrm{~mm}$ en el caso de los no infectados por $\mathrm{VIH}$ vacunados con $\mathrm{BCG}^{2,9}$. A todos aquellos sujetos no infectados se les repetía la prueba de la tuberculina con carácter anual, siguiendo el criterio anterior para diagnosticar la infección tuberculosa.

En el caso de los infectados por el bacilo tuberculoso y tras descartar la presencia de enfermedad se les explicó, de forma individualizada por el médico del centro, su situación, el riesgo de desarrollo de tuberculosis con base a su situación clínica (infección VIH, lesiones fibróticas, conversión reciente, etc.), la importancia de llevar a cabo el TIT con INH y los posibles efectos secundarios e indeseables del tratamiento. En los casos de previsible corta estancia en prisión no se indicó iniciar el tratamiento en el centro, derivándose a su médico de cabecera. En los sujetos mayores de 35 años que no eran ni convertores ni contactos con casos bacilares, así como en los sujetos con hepatopatía con niveles elevados de transaminasas (superiores a 200 UI) tampoco se indicó el TIT.

Los esquemas de tratamiento que se propusieron a los pacientes fueron los siguientes ${ }^{9}$ :

- $300 \mathrm{mgr} /$ día durante seis meses en el caso de no infectados por VIH y con radiografía normal.

- $300 \mathrm{mgr} / \mathrm{d}$ ra durante doce meses en el caso de infectados por VIH o con radiografía con formas residuales y/o fibróticas. 
PREVALENCIA DE TRATAMIENTO DE LA INFECCIÓN TUBERCULOSA EN UNA PRISIÓN PROVINCIAL

- $900 \mathrm{mgr} / \mathrm{día}$ en pauta bisemanal, durante seis o doce meses siguiendo los criterios anteriores.

Se ofrecía la posibilidad de seguir el tratamiento de forma directamente observada o bien en entrega semanal de la medicación tras la presentación de una receta estandarizada con la prescripción médica. En el caso de pacientes en programa de mantenimiento con metadona se les ofreció la dilución de INH liquida en la metadona.

De forma consensuada se intentaba elegir la modalidad de tratamiento que menos afectara a la vida ordinaria del paciente y era éste quien elegía entre las diversas opciones.

A los pacientes con TIT en el momento del estudio se procedió a tomar una única muestra de orina para la realización del test de EidusHamilton ${ }^{10}$. Sin previo aviso, se informaba al paciente en el momento de la toma de la muestra del objeto de la misma y se solicitaba su autorización.

\section{RESULTADOS}

El número total de internos ingresados en el CP el día 28 de junio era de 236 , de los cuales, $17(7,2 \%)$ habían realizado quimioterapia o tratamiento de la infección tuberculosa con anterioridad al ingreso en prisión.

Los 219 internos restantes eran mayoritariamente varones $(93,2 \%)$ con una edad media de 32,9 años $\pm 8,6$ años. El $34,7 \%$ eran o habían sido usuarios de drogas intravenosas, el $10,5 \%$ estaban infectados por el virus de la inmunodeficiencia humana y el $35,6 \%$ presentaban cicatriz post-vacunal por BCG (tabla 1). El 58\% (127) (I.C. 95\% = 64,5-51,5) cumplían criterios para ser clasificados como infectados por $M$. tuberculosis.

De los 127 sujetos infectados no se indicó TIT en $14(11 \%)$ debido a una previsible poca estancia en prisión (10), pacientes mayores de 35 años (3) y hepatopatía (1). De los 113 pacientes en los que se indicó TIT, $29(25,7 \%)$ no aceptaron el tratamiento propuesto. De los 84 casos en los que fue prescrito y aceptado el
Tabla 1

Características de la población sin antecedentes de tratamiento de enfermedad ni infección tuberculosa, ingresada en un Centro Penitenciario el día 28 de Junio de 1999 ( $N=219)$

\begin{tabular}{|lcc|}
\hline Variable & $N$ & $\%$ \\
\hline Infección tuberculosa & 127 & 58,0 \\
Varones & 204 & 93,2 \\
Usuarios de drogas intravenosas & 76 & 34,7 \\
Infectados por VIH & 23 & 10,5 \\
Vacunados con BCG & 78 & 35,6 \\
\hline
\end{tabular}

TIT, $22(26,2 \%)$, abandonaron el mismo antes de finalizar un curso completo (el $91 \%$ en el primer mes de iniciar el tratamiento) el $90 \%$ por «incomodidad», en dos casos por intolerancia digestiva y un caso por elevación de transaminasas y síndrome general. De los 62 pacientes restantes $39(62,9 \%)$ habían finalizado un curso completo de TIT y $23(37,1 \%)$ se encontraban realizando la misma en el momento del estudio (tabla 2).

De los 23 pacientes que se encontraban realizando TIT, 10 era con solución en metadona, 2 en TDO diaria y 1 en TDO bisemanal (56,5\% de los pacientes en TIT estaban en TDO). 0tros 10 pacientes más se encontraban en pauta diaria no directamente observada.

De los 23 pacientes, 5 tenían prescrito tratamiento de 12 meses, 3 por ser infectados por VIH y 2 por presentar radiografía con imágenes residuales. El total de pacientes en TIT llevaban una media de 88 días de tratamiento $(\mathrm{DE}=70$ días; Mediana $=83$ días; $\mathrm{P} 25-75=131$ días). Todos los pacientes aceptaron la realización del test de Eidus-Hamilton y en 22 pacientes $(95,7 \%)$ resultó positivo, sólo en el caso de un paciente en TIT no directamente observado resultó negativo. De los 127 sujetos infectados por M. tuberculosis, 61 (48\%, I.C. $95 \%=39,3-56,7$ ) o habian finalizado el TIT o estaban realizando correctamente el mismo. 
Tabla 2

Distribución del acceso y la adherencia al tratamiento de la infección tuberculosa en la población ingresada en un Centro Penitenciario el día 28 de Junio de 1999 sin antecedentes de tratamiento de enfermedad ni infección tuberculosa

\begin{tabular}{|lcc|}
\hline & N/Total & $\%$ \\
\hline Infectados por M. tuberculosis & $127 / 219$ & 58,0 \\
Se Indicó Tratamiento para la Infección Tuberculosa & $113 / 127$ & 89,0 \\
Iniciaron Tratamiento para la Infección Tuberculosa & $84 / 113$ & 74,3 \\
Habían finalizado Tratamiento para la Infección Tuberculosa & $39 / 84$ & 46,4 \\
Se encontraban realizando Tratamiento para la Infección Tuberculosa & $23 / 84$ & 27,4 \\
Test de Eidus-Hamilton positivo & $22 / 23$ & 95,7 \\
\hline
\end{tabular}

\section{DISCUSIÓN}

La prevalencia de infección tuberculosa $(58 \%)$ es muy superior a la observada en este mismo centro al ingreso en prisión $(37 \%)^{11}$. Esto puede deberse a las diferentes características socio-demográficas entre quienes ingresan en prisión y quienes permanecen más tiempo en la misma, al efecto booster por la repetición de test tuberculínicos y también al riesgo de transmisión de la infección tuberculosa en prisión. Éste mismo hecho ha sido observado en Estados Unidos en diversas instituciones de larga estancia, como asilos y prisiones $^{12}$. En una encuesta realizada el día 22 de junio de 1998 sobre una muestra de población reclusa ingresada en los Centros Penitenciarios españoles se observó una prevalencia de $50,6 \%$, si bien en un $38 \%$ de los pacientes se desconocía el estado en aquel momento de la infección tuberculosa por no haber realizado el seguimiento tuberculínico en los no infectados ${ }^{13}$.

El porcentaje de pacientes que no aceptaron TIT fue elevado $(25,7 \%)$ pero similar al observado en población reclusa americana donde el $27 \%$ no iniciaron TIT a pesar de que en muchos Estados este tratamiento es de carácter obligatorio ${ }^{14}$. La actividad desarrollada para conseguir el acceso al TIT era el consejo médico individualizado, lo que en muchas ocasiones no se mostró suficiente para conseguir el inicio del tratamiento, en casi todos los casos por un problema de creencias y actitudes que podría ser modificado con programas de marketing sanitario o incentivos ${ }^{7,8,15}$.

El $26,2 \%$ de abandonos fue similar al encontrado en población general en nuestro país $(31 \%)$ si bien en este caso había un importante porcentaje de mujeres y niños, habitualmente mejores cumplidores, y en todos los casos la prescripción de TIT fue de 6 meses ${ }^{5}$.

En el momento del estudio habían finalizado el tratamiento o se encontraban realizándolo correctamente el $48 \%$ de los infectados. Esto supone una amplia cobertura sobre el total de población ingresada susceptible de realizar el TIT, lo que tendrá evidente repcrcusión en la disminución de la incidencia de infección TB en las personas ingresadas. En EE.UU el $56 \%$ de los pacientes que iniciaron TIT lo finalizaron, si bien este estudio es de carácter longitudinal y no de corte transversal como en este caso ${ }^{14}$.

Otros autores encuentran que un porcentaje elevado (alrededor del 60\%) de aquellos que estaban realizando TIT no lo cumplían adecuadamente, bien demostrado a través de test de Eidus-Hamilton6 o por recuentos de recogida de INH en farmacia ${ }^{15}$. El alto porcentaje de cumplidores en nuestro estudio se puede deber 
en primer lugar a que se realizó una única determinación, pero también a que la mayoría de los pacientes se encontraba en TDO y a la educación sanitaria personalizada realizada antes de la prescripción y durante todo el tratamiento por el personal sanitario del centro. Diversos autores han observado como la TDO, los incentivos y la inclusión en el Programa de Mantenimiento con Metadona mejoran la efectividad del TIT $^{16}$ y como la Educación Sanitaria constituye una de las estrategias más eficaces para aumentar el acceso y la adherencia al TIT ${ }^{17}$. El conocimiento, por parte de los pacientes de los aspectos epidemiológicos de la TB y la importancia de un buen cumplimiento del TIT pueden haber influido en los resultados obtenidos ${ }^{6}$.

Se puede concluir que se ha observado una muy elevada prevalencia de infección tuberculosa, por lo que la eficacia de los programas de prevención y control de la TB en este medio van a tener una gran trascendencia clínica y epidemiológica, tanto en población reclusa como en la población general. El TIT, dadas estas altas prevalencias de infección tuberculosa, se presenta como una necesaria estrategia que puede conseguir aceptables niveles de acceso y cumplimiento en este colectivo mediante el TDO y la Educación Sanitaria.

\section{BIBLIOGRAFÍA}

1. Drobniewski F. Tuberculosis in prison-forgotten plague. Lancet 1995; 346: 948-49.

2. Grupo de Trabajo sobre Tuberculosis. Consenso Nacional para el Control de la Tuberculosis en España. Med Clín (Barc) 1992; 98: 24-31.

3. Martín V, González P, Caylà JA, Mirabent J, Cañellas J, Pina JM, Miret P. Case-finding of pulmonary tuberculosis on admission to a penitentiary centre. Tuber Lung Dis 1994; 74: 49-53.

4. Wilkinson D, Squire SB, Garner P. Effect of preventive treatment for tuberculosis in adults infected with HIV: systematic review of randomised placebo control trials. BMJ 1998; 317: 625-629.

5. Galván Olivares F, Santiuste de Pablos C. Factores relacionados con el cumplimiento de la quimioprofilaxis contra la tuberculosis. Mcd Clin (Barc) 1998; 111: 655-657.
6. Romero Saldaña $M$, Vaquero Abellan $M$, Gallego Rubio R, Aguilera López MD, de Celis Cornejo JM, Barquín García E, et al. Valoración del cumplimiento de la QP antituberculosa por la población reclusa del Centro Penitenciario de Jaén. Rev Esp Salud Pública 1997; 71: 391-399.

7. Esteban A, Seral A, Castanera A. Estudio del cambio de actitudes en el Centro Penitenciario de Huesca respecto al programa de prevención de la tuberculosis. Libro de Ponencias y Comunicaciones. 348. II Congreso Nacional de Sanidad Penitenciaria. Barcelona; 1998.

8. Arroyo JM, Aso M, Fernández de la Hoz K, Otal F, Febrel M. Adherencia a la quimioprofilaxis antituberculosa. Empleo de técnicas de marketing sanitario y educación para la salud en el medio penitenciario. Rev Esp Sanid Penit. 1998; 1: 7-11.

9. Subdirección General de Sanidad Penitenciaria. Programa de Prevención y Control de la tuberculosis en Instituciones Penitenciarias. Madrid: Ministerio de Justicia; 1990.

10. Eidus L, Hamilton EJ. A new method for the detection of $\mathrm{N}$-acetyl isoniazid in urine of ambulatory patients. Am Rev Respir Dis. 1964; 89: 587-588.

11. Martín V, Caylà JA, Bolea A, de Paz JA. Evolución de la prevalencia de infección por Mycobacterium tuberculosis en población reclusa al ingreso en prisión. Med Clín (Barc) 1998; 111: 11-16.

12. Stead W. Special Problems in Tuberculosis: Tuberculosis in the Elderly and in Residents of Nursing Homes, Correctional Facilities, LongTerm Care Hospitals, Mental Hospitals, Shelters for the Homeless, and Jails. Clin Chest Med 1989; 10, 3: 397-405.

13. Subdirección General de Sanidad Penitenciaria. Encuesta 22-J. Madrid: Ministerio del Interior; 1998.

14. Lobato MN, Leary L, Hayden C, Simone PM. Completion of treatment for TB infection in Correctional Facilities, United States, 1992-1996. Abstract Book. 30th IUATLD World Conference on Lung Health. Madrid; 1999. S17.

15. Arroyo JM, Lacal P. Un programa de promoción de la salud en cl medio penitenciario. Trabajo Soc Salud 2000; 35: 499-503. 
16. Alcabes P, Vossenas P, Cohen R, Braslow C, Nichaels D, Zoloth S. Compliance with Isoniazid Prophilaxis in Jail. Am Rev Resp Dis 1989; 140 1194-1197.

17. Snyder DC, Paz EA, Mohle-Boetani JC, Fallstad R, Black RL, Chin DP Tuberculosis prevention in methadone maintenance clinics. Effectiveness and cost-effectiveness.
Am J Respir Crit Care Med 1999; 160 (1): 178-85.

18. Alcaide Megías $\mathrm{J}$, Altet Gómez $\mathrm{N}$, Canela Soler J, Serra Majen L, Garrido Morales P, Navas Alcalá MR, et al. Influencia de la educación sanitaria en el cumplimiento de la quimioprofilaxis antituberculosa en niños: ensayo comunitario. Rev Clin Esp 1990; 187: 89-93 